Pacific Journal of Mathematics

CONVEX INTEGRANDS ON SOUSLIN LOCALLY CONVEX 


\title{
CONVEX INTEGRANDS ON SOUSLIN LOCALLY CONVEX SPACES
}

\author{
M. VALADIER
}

\begin{abstract}
R. T. Rockafellar has proved a formula for the conjugates of convex integral functionals on vector spaces of measurable vector-valued functions. This formula is extended to the case where the spaces of values of the measurable functions is a Souslin locally convex space. Rockafellar's definition of decomposable space of measurable vector-valued functions is extended to other than normed spaces. In the first section technical results on measurable set-valued functions are given.
\end{abstract}

Introduction. Let $(T, \mathscr{C}, \mu)$ denote a measure space with a positive $\sigma$-finite measure $\mu$ and $\mathscr{C}$ complete. Let $E$ be a real locally convex space and $E^{\prime}$ its dual. Let $\mathscr{L}\left(\right.$ resp. $\left.\mathscr{L}^{\prime}\right)$ be a vector space of functions from $T$ to $E$ (resp. $E^{\prime}$ ). Hypotheses will be made which ensure that for each $u \in \mathscr{L}$ and each $v \in \mathscr{L}^{\prime}$, the function $t \mapsto\langle v(t)$, $u(t)\rangle$ is integrable. The pairing $\int\langle v(t), u(t)\rangle \mu(d t)$ will be denoted by $\langle v, u\rangle$.

A function $f: T \times E \rightarrow \overline{\boldsymbol{R}}(=[-\infty, \infty])$ will be called an integrand. Under certain hypotheses $t \mapsto f(t, u(t))$ is measurable for each $u \in \mathscr{L}$. We shall consider the functional on $\mathscr{L}$ defined by $I_{f}(u)=\int f(t, u(t)) \mu(d t)$ (with the convention that the integral is $+\infty$ if the positive part of $f(t, u(t))$ is nonintegrable). Denote by $f^{*}(t, \cdot)$ the conjugate function of $f(t, \cdot)$ this is

$$
f^{*}\left(t, x^{\prime}\right)=\sup \left\{\left\langle x^{\prime}, x\right\rangle-f(t, x) \mid x \in E\right\} \text { for } x^{\prime} \in E^{\prime} .
$$

As above $I_{f^{*}}$ denotes the functional on $\mathscr{L}^{\prime}$ defined by $I_{f^{*}}(v)=$ $\int f^{*}(t, v(t)) \mu(d t)$.

Then roughly speaking the result proved by Rockafellar is the following: if $\mathscr{L}$ contains sufficiently many functions (see below the definition of decomposable spaces) then $I_{f *}$ is the conjugate functional of $I_{f}$ with respect to the duality $\mathscr{L}, \mathscr{L}^{\prime}$.

More precisely, for each $v \in \mathscr{L}^{\prime}$,

$$
I_{f *}(v)=\left(I_{f}\right)^{*}(v)\left(=\sup \left\{\langle v, u\rangle-I_{f}(u) \mid u \in \mathscr{L}\right\}\right)
$$

Rockafellar first proved this formula for $E=\boldsymbol{R}^{n}$ ([11] Th. 2 p. 532), then for separable Banach spaces ([13] Th. 2 p. 225). The inequality $I_{f *}(v) \geqq\left(I_{f}\right)^{*}(v)$ is obvious. The converse inequality is proved using measurable selection theorems (and the fact that $\mathscr{L}$ contains sufficiently many functions). Standard measurable selection theorems 
require Polish spaces. But if $E$ is a separable Banach space its dual may fail to be Polish, and then it is difficult to prove $\left(I_{f *}\right)^{*}=I_{f * *}$.

In [5] (Theorem 5) Castaing proved the two formulas $\left(I_{f}\right)^{*}=I_{f *}$ and $\left(I_{f *}\right)^{*}=I_{f^{* *}}$ for $E$ separable Fréchet but $T$ metrizable compact. In [6] he succeeds to prove the same formulas for abstract $T$ and $E$ separable Banach space, using the fact that $E^{\prime}$ endowed with $\sigma\left(E^{\prime}, E\right)$ is a Lusin space.

Here we shall extend the formulas to a Souslin locally convex space $E$ whose dual $E^{\prime}$ is also a Souslin space for at least one locally convex topology compatible with duality. That is the case if $E$ is a separable Fréchet space, and many locally convex spaces deduced from separable Fréchet spaces (for example most of the spaces encountered in the theory of distributions) have that property. Some new results on measurable functions and measurable set-valued functions with values in a Souslin space are given in a preliminary section. In particular Lemma 2 extends Bourbaki ([2] Ch. IV § 5). For applications and further references see Ioffe-Levin [7], IoffeTikhomirov [8], Rockafellar [12].

1. Functions and set-valued functions with values in a Souslin space. We shall denote by $\mathscr{B}(E)$ the Borel $\sigma$-field of a topological space $E$. A Souslin space is a Hausdorff topological space $S$ such that there exists a Polish space $P$ and a continuous map $h$ from $P$ onto $S$.

Lemma 1. Let $S$ be a Souslin space, $P$ a Polish space and $h$ a continuous map from $P$ onto $S$. Let $\Gamma$ be a set-valued function from $T$ to the closed nonempty subsets of $S$, whose graph belongs to $\mathscr{C} \otimes \mathscr{B}(S)$. Then

(a) $\Gamma$ admits a sequence of selections $\left(u_{n}\right)$, such that, for every $t$ the $u_{n}(t)$ are dense in $\Gamma(t)$, and such that there exist measurable maps $\sigma_{n}: T \rightarrow P$ with $u_{n}=h \circ \sigma_{n}$.

(b) Moreover $u_{n}$ has the following properties:

1. $u_{n}$ is $\left(\mathscr{C}, \mathscr{B}(S)\right.$ ) measurable (that is $\forall A \in \mathscr{B}(S), u_{n}^{-1}(A) \in \mathscr{C}$ )

2. $u_{n}$ is the limit of a sequence of $\mathscr{C}$-measurable functions assuming a finite number of values.

3. Moreover if $T$ is a Hausdorff topological space, and $\mu$ a Radon measure, then $u_{n}$ is Lusin $\mu$-measurable.

Proof. (a) Let $G$ denote the graph of $\Gamma$ and $\mathrm{pr}_{T}$ denote the map $(t, x) \mapsto t$ from $T \times S$ to $T$. Put $\phi(t)=h^{-1}(\Gamma(t))$. It is a closed nonempty subset of $P$. Recall that if $U$ is a subset of $P, \phi^{-}(U)$ denotes the set $\{t \in T \mid \phi(t) \cap U \neq \varnothing\}$.

Then if $U \in \mathscr{B}(P)$ one has 


$$
\begin{aligned}
\phi^{-}(U) & =\{t \mid \phi(t) \cap U \neq \varnothing\} \\
& =\{t \mid \Gamma(t) \cap h(U) \neq \varnothing\} \\
& =\Gamma^{-}(h(U)) \\
& =\operatorname{pr}_{T}(G \cap(T \times h(U)) .
\end{aligned}
$$

It is easy to see that $G \cap(T \times h(U))$ belongs to $\mathscr{C} \otimes \mathscr{B}(h(U))$. But $h(U)$ is a Souslin space (because $U$ is Borel hence Souslin). Thus by the projection theorem (Aumann [1], Sainte Beuve [14]; for completeness we sketch a short proof in the Remark 2 below) $\phi^{-}(U)$ belongs to $\mathscr{C}$. Thus we can apply standard theorems on measurable selections: Castaing ([4] Th. 5.2) and for abstract measurability Valadier ([16] Th. 0.3). The conclusion is: $\phi$ has a sequence of measurable selections $\left(\sigma_{n}\right)$ such that for every $t$ the $\sigma_{n}(t)$ are dense in $\phi(t)$. Put $u_{n}(t)=h \circ \sigma_{n}(t)$. Then the $u_{n}(t)$ are dense in $\Gamma(t)$.

(b) 1. As $\sigma_{n}$ is $(\mathscr{C}, \mathscr{B}(P))$ measurable and $h$ is continuous (hence $\left(\mathscr{B}(P), \mathscr{B}(S)\right.$ ) measurable), $u_{n}$ is $(\mathscr{C}, \mathscr{B}(S))$ measurable.

2. The function $\sigma_{n}$ is the limit of a sequence of $\mathscr{C}$-measurable functions assuming a finite number of values. Hence $u_{n}$ has the same property.

3. Finally if $T$ is a Hausdorff topological space and $\mu$ a Radon measure (see Bourbaki [3] Schwartz [15] for measures on Hausdorff spaces), it is well known that, as $P$ is Polish, $\sigma_{n}$ is Lusin measurable. That is for each compact $K \subset T$ and $\varepsilon>0$, there exists a compact $K_{\varepsilon} \subset K$ such that $\mu\left(K-K_{\varepsilon}\right) \leqq \varepsilon$ and $\sigma_{n}$ is continuous on $K_{\varepsilon}$. Obviously $u_{n}$ has the same property.

\section{REMARKS.}

(1) Existence of one measurable selection has been proved by Sainte-Beuve [14] under a weaker hypothesis: $\Gamma$ is not supposed closed-valued. She extends Aumann's theorem, which was stated for a Lusin space.

(2) We sketch now a short proof of the projection theorem. The statement is the following: if $S$ is Souslin and $G \in \mathscr{C} \otimes \mathscr{B}(S)$, the projection of $G$ onto $T$ belongs to $\mathscr{C}$. When $S$ is compact metrizable, this theorem is well known (Meyer [9], Neveu [10]): $G$ is analytic and its projection is analytic, hence belongs to $\mathscr{C}$ which has been supposed complete.

If $S$ is Polish, $S$ is $G_{\delta}$ (countable intersection of open sets) in a compact metrizable space $E$. Then it is obvious that $\mathscr{C} \otimes \mathscr{B}(S) \subset$ $\mathscr{C} \otimes \mathscr{B}(E)$ and the projection theorem is true for $S$ Polish. Finally if $S$ is Souslin: let $P$ be a Polish space and $h: P \rightarrow S$ continuous and onto. Then if $G \in \mathscr{C} \otimes \mathscr{B}(S),\left(1_{T} \times h\right)^{-1}(\mathrm{G})$ belongs to $\mathscr{C} \otimes \mathscr{B}(P)$ and 


$$
\operatorname{pr}_{T} G=\operatorname{pr}_{T}\left[\left(1_{T} \times h\right)^{-1}(G)\right] .
$$

Here $1_{T} \times h$ is the map $(t, x) \mapsto(t, h(x))$ from $T \times P$ to $T \times S$, and $\mathrm{pr}_{T}$ denotes either the projection from $T \times S$ onto $T$ or the projection from $T \times P$ onto $T$.

CoRollary. If $S$ is a Souslin space and $u: T \rightarrow S$ is a function whose graph belongs to $\mathscr{C} \otimes \mathscr{B}(S)$, then u has the following properties:

(1) $u$ is $(\mathscr{C}, \mathscr{B}(S))$ measurable

(2) $u$ is the limit of a sequence of $\mathscr{C}$-measurable functions assuming a finite number of values

(3) Moreover if $T$ is a Hausdorff topological space, and $\mu a$ Radon measure, then $u$ is Lusin $\mu$-measurable.

Proof. Apply Lemma 1(b) to $\Gamma(t)=\{u(t)\}$.

LEMMA 2. Let $E$ be a Souslin real locally convex vector space, and $u: T \rightarrow E$ a function.

(a) Then the four following properties are equivalent:

(1) $u$ is $(\mathscr{C}, \mathscr{B}(E))$ measurable

(2) $u$ is the limit of a sequence of C-measurable functions assuming a finite number of values

(3) $u$ is scalarly measurable (that is for each $x^{\prime} \in E^{\prime},\left\langle x^{\prime}, u(\cdot)\right\rangle$ is measurable)

(4) the graph of $u$ belongs to $\mathscr{C} \otimes \mathscr{B}(E)$.

(b) Moreover if $T$ is a Hausdorff topological space, and $\mu a$ Radon measure, consider the property

(5) $u$ is Lusin $\mu$-measurable.

Then the five properties (1), .., (5) are equivalent.

Proof. (a) The corollary to Lemma 1 yields the implications $4 \Rightarrow 1$ and $4 \Rightarrow 2$. $1 \Rightarrow 3$ and $2 \Rightarrow 3$ are obvious. We prove now $3 \Rightarrow 4$. By Lemma 3 below, there exists a sequence $\left(e_{n}^{\prime}\right)$ in $E^{\prime}$ which separates points of $E$. Thus the graph of $u$ is

$$
\bigcap_{n}\left\{(t, x) \in T \times E \mid\left\langle e_{n}^{\prime}, x\right\rangle=\left\langle e_{n}^{\prime}, u(t)\right\rangle\right\} .
$$

Hence if $u$ is scalarly measurable, the graph of $u$ belongs to $\mathscr{C} \otimes$ $\mathscr{B}(E)$.

(b) Suppose that $T$ is a Hausdorff topological space and that $\mu$ is Radon. Then $5 \Rightarrow 3$ is obvious and $4 \Rightarrow 5$ is the corollary of Lemma 1.

Lemma 3. Let $S$ be a Souslin space and $\left(f_{i}\right)_{i \in I}$ a family of real-valued continuous functions which separates points of $S$ (that 
is if $x \neq y$, there exists $i$ such that $\left.f_{i}(x) \neq f_{i}(y)\right)$, then there exists $a$ countable subset $D$ of $I$ such that the subfamily $\left(f_{i}\right)_{i \in D}$ separates points of $S$.

Proof. The fact that $\left(f_{i}\right)_{i \in I}$ separates points of $S$ is equivalent to

$$
S^{2}-\Delta_{S}=\bigcup_{i \in I}\left(f_{i} \times f_{i}\right)^{-1}\left(\boldsymbol{R}^{2}-\Delta_{R}\right) .
$$

In this formula $f_{i} \times f_{i}$ denotes the map $(x, y) \mapsto\left(f_{i}(x), f_{i}(y)\right)$, and $\Delta_{E}$ denotes the diagonal in $E \times E$. As $S^{2}$ is Souslin, there exists a Polish space $Q$ and a continuous onto map $k: Q \rightarrow S^{2}$. Put

$$
U_{i}=\left(f_{i} \times f_{i}\right)^{-1}\left(\boldsymbol{R}^{2}-\Delta_{R}\right) \text {. }
$$

It is an open set. It is well known that there exists a countable subset $D$ of $I$ such that

$$
\bigcup_{i \in D} k^{-1}\left(U_{i}\right)=\bigcup_{i \in I} k^{-1}\left(U_{i}\right)
$$

As $k$ is onto, that implies

$$
\bigcup_{i \in D} U_{i}=\bigcup_{i \in I} U_{i}
$$

Hence the countable subfamily $\left(f_{i}\right)_{i \in D}$ separates points of $S$.

REMARK. This result has been proved by Schwartz [15] in a more general form.

Lemma 4. Let $E$ be a Souslin locally convex space and $u: T \rightarrow E$ scalarly measurable. Then the function $\left(t, x^{\prime}\right) \mapsto\left\langle x^{\prime}, u(t)\right\rangle$ defined on $T \times E^{\prime}$, is $\mathscr{C} \otimes \mathscr{B}\left(E^{\prime}\right)$ measurable.

Proof. This follows from property (2) of Lemma 2.

Indeed let $u=\lim u_{n}$ where the $u_{n}$ are $\mathscr{C}$-measurable functions assuming a finite number of values. Then $u_{n}(t)=x_{n}^{p}$ if $t \in T_{n}^{p}$, and

$$
\left\langle x^{\prime}, u_{n}(t)\right\rangle=\left\langle x^{\prime}, x_{n}^{p}\right\rangle \quad \text { if } \quad t \in T_{n}^{p} .
$$

Thus $\left(t, x^{\prime}\right) \mapsto\left\langle x^{\prime}, u_{n}(t)\right\rangle$ is $\mathscr{C} \otimes \mathscr{B}\left(E^{\prime}\right)$ measurable on $T_{n}^{p} \times E^{\prime}$, hence on all $T \times E^{\prime}$. Finally $\left\langle x^{\prime}, u(t)\right\rangle=\lim \left\langle x^{\prime}, u_{n}(t)\right\rangle$ is a $\mathscr{C} \otimes \mathscr{B}\left(E^{\prime}\right)$ measurable function of $\left(t, x^{\prime}\right)$.

LEMma 5. Let $E$ be a Souslin locally convex space and $u: T \rightarrow E$ scalarly measurable. Then there exists a sequence $\left(T_{n}\right)$ in $\mathscr{C}$ such that $\overline{u\left(T_{n}\right)}$ is compact, and $T-\cup T_{n}$ is $\mu$-negligible. 
Proof. As $\mu$ is $\sigma$-finite it is sufficient to prove the result when $\mu$ is bounded. By property (1) of Lemma 2 one may consider the measure $\nu=\mu \circ u^{-1}$ on $(E, \mathscr{B}(E))$. As $E$ is Souslin, $\nu$ is a Radon measure (Bourbaki [3] Prop. 3 p. 49). Therefore there exists a sequence of compact sets $\left(K_{n}\right)$ in $E$, such that $\nu\left(\cup K_{n}\right)=\nu(E)$. The sets $T_{n}=u^{-1}\left(K_{n}\right)$ have the required properties.

2. Decomposable vector spaces of functions. Integrands. From now on $E$ is a Souslin real locally convex vector space and its dual $E^{\prime}$ is supposed to be Souslin for at least one topology compatible with duality (we remark that this is equivalent to supposing that $E^{\prime}$ is Souslin for the weak topology $\sigma\left(E^{\prime}, E\right)$ ).

We denote by $\mathscr{L}$ (resp. $\left.\mathscr{L}^{\prime}\right)$ a vector space of scalarly measurable functions from $T$ to $E$ (resp. $E^{\prime}$ ), and by $L$ (resp. $L^{\prime}$ ) the space of equivalence classes for equality almost everywhere. Note that by property (2) of Lemma 2 for each $u \in \mathscr{L}$, and each $v \in \mathscr{L}^{\prime}, t \mapsto\langle v(t)$, $u(t)\rangle$ is measurable. We make the hypothesis that for each $u \in \mathscr{L}$ and each $v \in \mathscr{L}^{\prime}, t \mapsto\langle v(t), u(t)\rangle$ is integrable. We denote by $\langle v, u\rangle$ the number $\int\langle v(t), u(t)\rangle \mu(d t)$. We denote by $\mathscr{M}_{E}^{k}$ (resp. $\left.\mathscr{M}_{E^{\prime}}^{k}\right)$ the space of scalarly measurable functions from $T$ to $E$ (resp. $E^{\prime}$ ) such that $\overline{f(T)}$ is compact (here it is important to choose a Souslin topology on $\left.E^{\prime}\right)$.

Definition 1. The space $\mathscr{L}$ is said to be decomposable if $u \in \mathscr{L}, f \in \mathscr{M}_{E}^{k}, A \in \mathscr{C}$ and $\mu(A)<\infty$ imply

$$
\chi_{A} f+\chi_{T-A} u \in \mathscr{L}
$$

$\left(\chi_{A}\right.$ denotes the characteristic function of $A$ ).

REMARK. If $E$ is a separable reflexive Banach space, then $E$ and $E^{\prime}$ are Polish for the norm topology, hence Souslin for all topologies compatible with duality. Our definition is equivalent to Rockafellar's (where $\mathscr{L}_{E}^{\infty}$ is taken in place of $\mathscr{M}_{E}^{k}$ ).

ExAmple. Let $E$ be a separable Fréchet space. Then its dual $E_{s}^{\prime}$ with the topology $\sigma\left(E^{\prime}, E\right)$ is Souslin. We may take $\mathscr{L}=\mathscr{L}_{E}^{1}$ and $\mathscr{L}^{\prime}=\mathscr{L}_{E_{\dot{\xi}}}^{\infty}$. Indeed, $\mathscr{L}_{E}^{1}$ is obviously decomposable. And $\mathscr{L}_{E_{s}^{\prime}}^{\infty}=\mathscr{M}_{E_{s}^{\prime}}^{k}$, because for a closed subset of $E_{s}^{\prime}$ compactness is equivalent to equicontinuity. Thus $\mathscr{L}_{E_{s}^{\prime}}^{\infty}$ is decomposable.

LEMMA 6.

(1) If $\mathscr{L}$ is decomposable and $v \in \mathscr{L}^{\prime}$, then $\forall u \in \mathscr{L},\langle v, u\rangle=0$, implies $v=0$ a.e. 
(2) If $\mathscr{L}$ and $\mathscr{L}^{\prime}$ are decomposable, the bilinear map $(u, v) \mapsto$ $\langle v, u\rangle$ defines a separated duality between $L$ and $L^{\prime}$.

Proof. (1) Let $A \in \mathscr{C}$ with $\mu(A)<\infty$. If $x \in E$ and $\varphi$ is a real valued bounded measurable function, then $\varphi x$ belongs to $\mathscr{M}_{E}{ }^{k}$. Hence $\chi_{A} \varphi x \in \mathscr{L}$. This entails $\int_{A}\langle v(t), x\rangle \varphi(t) \mu(d t)=0$, for each $x$ and each $\varphi$. Hence $\langle v(\cdot), x\rangle=0$ almost everywhere on $A$, hence on $T$. As $E$ is the dual of $E^{\prime}$, it contains a sequence $\left(e_{n}\right)$ which separates points of $E^{\prime}$ (Lemma 3). Therefore $v=0$ a.e.

(2) The second part is obvious from the first.

Definition 2. A function $f: T \times E \rightarrow \bar{R}(=[-\infty, \infty])$ is said to be a normal integrand on $T \times E$ if for every $t, f(t, \cdot)$ is lower semicontinuous and $f$ is $\mathscr{C} \otimes \mathscr{B}(E)$ measurable. It is said to be a convex normal integrand if it is a normal integrand and for every $t, f(t, \cdot)$ is convex.

In the following lemma epi $f(t, \cdot)$ denotes

$$
\{(x, r) \in E \times \boldsymbol{R} \mid r \geqq f(t, x)\} .
$$

LEMMA 7. The function $f$ is a normal integrand iff the setvalued function $t \mapsto$ epi $f(t, \cdot)$ is closed valued and its graph belongs to $\mathscr{C} \otimes \mathscr{B}(E) \times \mathscr{B}(\boldsymbol{R})$.

Proof. First note that the closure of epi $f(t, \cdot)$ is equivalent to lower semi-continuity of $f(t, \cdot)$.

(1) Suppose $f$ is a normal integrand. The graph $G$ of $t \mapsto \operatorname{epi} f(t, \cdot)$ is given by the formula

$$
G=\{(t, x, r) \mid r \geqq f(t, x)\}
$$

and hence belongs to $\mathscr{C} \otimes \mathscr{B}(E) \otimes \mathscr{B}(\boldsymbol{R})$.

(2) Suppose that the graph $G$ belongs to $\mathscr{C} \otimes \mathscr{B}(E) \otimes \mathscr{B}(R)$. Therefore, for each $r \in \boldsymbol{R},\{(t, x) \mid(t, x, r) \in G\}$ belongs to $\mathscr{C} \otimes \mathscr{B}(E)$ (Neveu [10] Prop. III-1-2). But

$$
\{(t, x) \mid(t, x, r) \in G\}=\{(t, x) \mid f(t, x) \leqq r\} .
$$

Thus $f$ is $\mathscr{C} \otimes \mathscr{B}(E)$ measurable.

REMARK. It is easy to see (using the fact that $R$ has a countable basis of open sets) that $\mathscr{B}(E) \otimes \mathscr{B}(R)=\mathscr{B}(E \times R)$.

LEMMA 8. If $f$ is a normal integrand on $T \times E$, then the function defined by 


$$
f^{*}\left(t, x^{\prime}\right)=\sup \left\{\left\langle x^{\prime}, x\right\rangle-f(t, x) \mid x \in E\right\}
$$

is a convex normal integrand on $T \times E^{\prime}$.

Proof. By Lemma 7 (and the remark) the set-valued function $t \mapsto$ epi $f(t, \cdot)$ has a measurable graph. By Lemma 1 there exists a sequence of measurable selections $\left(u_{n}, r_{n}\right)$ such that for every $t$ the $\left(u_{n}(t), r_{n}(t)\right)$ are dense in epi $f(t, \cdot)$.

Thus by Lemma 4

$$
f^{*}\left(t, x^{\prime}\right)=\sup _{n}\left[\left\langle x^{\prime}, u_{n}(t)\right\rangle-r_{n}(t)\right]
$$

is a measurable function of $\left(t, x^{\prime}\right)$.

\section{Conjugate integral functionals.}

Definition 3. Let $f$ be a normal integrand on $T \times E$. An integral functional is defined on $\mathscr{L}$ by

$I_{f}(u)=\int_{T} f(t, u(t)) \mu(d t)$, with the convention $(+\infty)+(-\infty)=$ $+\infty$, that is the integral is $+\infty$ if positive and negative parts of $f(t, u(t))$ are nonintegrable.

THeOREM. If $\mathscr{L}$ is decomposable, if there exists $u_{0} \in \mathscr{L}$ such that $I_{f}\left(u_{0}\right)<\infty$, then $I_{f *}$, is the polar functional of $I_{f}$, that is, for every $v \in \mathscr{L}^{\prime}$

$$
I_{f *}(v)=\sup \left\{\langle v, u\rangle-I_{f}(u) \mid u \in \mathscr{L}\right\} .
$$

If in addition $f$ is convex, $\mathscr{L}^{\prime}$ decomposable and $I_{f *}\left(v_{0}\right)<\infty$ for at least one $v_{0} \in \mathscr{L}^{\prime}$, then $I_{f}$ and $I_{f^{*}}$ are mutually convex lower semicontinuous polar functional on $\mathscr{L}$ and $\mathscr{L}^{\prime}$.

Proof. The proof follows Rockafellar [13].

We can rewrite the formula

$$
\begin{aligned}
\int \sup & \{\langle v(t), x\rangle-f(t, x) \mid x \in E\} \mu(d t) \\
& =\sup \left\{\int\langle v(t), u(t)\rangle \mu(d t)-\int f(t, u(t)) \mu(d t) \mid u \in \mathscr{L}\right\} .
\end{aligned}
$$

(If we rewrite the second member

$$
\sup \left\{\int[\langle v(t), u(t)\rangle-f(t, u(t))] \mu(d t) \mid u \in \mathscr{L}\right\}
$$

we have to use the opposite convention, that $-\infty$ prevails over $+\infty$.) Thus the inequality $\geqq$ is obvious. To prove $\leqq$, let $\beta \in \boldsymbol{R}$ such 
that $\beta<I_{f *}(v)$ and let us find $u$ such that $\langle v, u\rangle-I_{f}(u) \geqq \beta$.

(1) As $I_{f}\left(u_{0}\right)<\infty$ there exists $\alpha_{0} \in \mathscr{L}^{1}$ such that $\left\langle v(t), u_{0}(t)\right\rangle-$ $f\left(t, u_{0}(t)\right) \geqq \alpha_{0}(t)$ a.e. (for example one can take $\alpha_{0}(t)=\left\langle v(t), u_{0}(t)\right\rangle-$ $\left.f^{+}\left(t, u_{0}(t)\right)\right)$. Remark that $f^{*}(t, v(t)) \geqq \alpha_{0}(t)$.

(2) Now we prove that there exists $\alpha_{1} \in \mathscr{L}^{1}$ such that $\int \alpha_{1}(t) \mu(d t)>\beta$ and $\alpha_{1}(t)<f^{*}(t, v(t))$ a.e. Indeed let $h \in \mathscr{L}^{1}$ have strictly positive finite values (we recall that $\mu$ is $\sigma$-finite). If $I_{f *}(v)<\infty$ put $\alpha_{1}(t)=f^{*}(t, v(t))-\varepsilon h(t)$ with $\varepsilon>0$ sufficiently small.

If $I_{f *}(v)=+\infty$ put

$$
\xi_{n}(t)=\left\{\begin{array}{l}
\inf \left(n h(t), \frac{1}{2} f^{*}(t, v(t))\right) \text { if } f^{*}(t, v(t))>0 \\
f^{*}(t, v(t))-h(t) \text { if } f^{*}(t, v(t)) \leqq 0
\end{array}\right.
$$

Then $\xi_{n} \in \mathscr{L}^{1},\left(\xi_{n}\right)$ is increasing, and $\xi_{n}(t) \rightarrow(1 / 2) f^{*}(t, v(t))$ if $f^{*}(t, v((t)>0$. By the monotone convergence theorem $\int \xi_{n} \mu \rightarrow \infty$. Choose $n$ large enough such that $\int \xi_{n} \mu>\beta$ and put $\alpha_{1}=\xi_{n}$. In each of the three cases $f^{*}(t, v(t))=+\infty$, finite $>0$ or $\leqq 0$, one has $\alpha_{1}(t)<f^{*}(t, v(t))$.

(3) Let $\Gamma(t)=\left\{x \in E \mid\langle v(t), x\rangle-f(t, x) \geqq \alpha_{1}(t)\right\}$.

It is a closed almost everywhere nonempty set. The graph of $\Gamma$ is

$$
\left\{(t, x) \mid\langle v(t), x\rangle-f(t, x) \geqq \alpha_{1}(t)\right\}
$$

and therefore belongs to $\mathscr{C} \otimes \mathscr{B}(E)$. Then (Lemma 1) $\Gamma$ has a measurable selection $u_{1}$. By Lemma 5 there exists an increasing sequence $\left(T_{n}\right)$ in $\mathscr{C}$ such that

$$
\begin{aligned}
& -\mu\left(T_{n}\right)<\infty \\
& -T-\cup T_{n} \text { is negligible } \\
& -\overline{u_{1}\left(T_{n}\right)} \text { is compact. }
\end{aligned}
$$

For $n$ large enough one has

$$
\int_{T_{n}} \alpha_{1} \mu+\int_{T-T_{n}} \alpha_{0} \mu \geqq \beta
$$

Put

$$
u(t)=\left\{\begin{array}{lll}
u_{1}(t) & \text { if } & t \in T_{n} \\
u_{0}(t) & \text { if } & t \in T-T_{n}
\end{array}\right.
$$

Then $u \in \mathscr{L}$ because $\mathscr{L}$ is decomposable.

On $T_{n}$ one has

$$
\langle v(t), u(t)\rangle-f(t, u(t)) \geqq \alpha_{1}(t)
$$

and on $T-T_{n}$ 


$$
\langle v(t), u(t)\rangle-f(t, u(t)) \geqq \alpha_{0}(t)
$$

\section{Hence}

$$
\int_{T}\langle v(t), u(t)\rangle \mu(d t)-\int_{T} f(t, u(t)) \mu(d t) \geqq \int_{T} \alpha_{1} \mu+\int_{T-T_{n}} \alpha_{0} \mu \geqq \beta .
$$

(Note that $f^{+}(t, u(t))$ is integrable so that $\int f(t, u(t)) \mu(d t)$ is not $+\infty$.) That proves the inequality $\leqq$. The remainder of the theorem is obvious.

\section{REFERENCES}

1. R. J. Aumann, Measurable utility and measurable choice theorem, Proceedings of the Colloque International du C.N.R.S. "La Décision". Aix en Provence, 1967. p. 15-26. 2. N. Bourbaki, Intégration Ch. I-IV, 2ème édition.

3. - Intégration Ch. IX.

4. CH. Castaing, Sur les multi-applications measurables, Thèse. Caen 1967 largely contained in Revue Inf. Rech. Op. (1967) 91-126.

5. — Quelques applications du théorème de Banach Dieudonné à l'intégration, Montpellier 1967-70 publication $\mathrm{n}^{\circ} 67$.

6. - Intégrales convexes duales, C. R. Acad. Sci., Paris 275 (1972), 1331-1334.

7. A. D. Ioffe and V. L. Levin, Subdifferential of convex functions Trudy, Mosk. Mat. Ob., 26 (1972), 3-73.

8. A. D. Ioffe and V. M. Tikhomirov, Duality of convex functions and extremum problems, Uspehi Mat. Nauk, 23 (1968), 51-116.

9. P. A. Meyer, Probabilités et Potentiel Hermann, 1966.

10. J. Neveu, Bases mathématiques du calcul des probabilités Masson, 1964.

11. R. T. Rockafellar, Integrals which are convex functionals, Pacific J. Math., 24 (1968), 525-539.

12. - Integrals which are convex functionals II, Pacific J. Math., 39 (1971), 439-469.

13. - Convex integral functionals and duality, Contributions to non linear functional analysis. Academic Press (1971) p. 215-236.

14. M. F. Sainte-Beuve, On the extension of von Neumann-Aumann's theorem, J. of Funct. Analysis, 17 (1974), 112-129.

15. L. Schwartz, Radon measures on topological spaces, Tata Institute.

16. M. Valadier, Contribution à l'analyse convexe Thèse Paris 1970.

Received May 10, 1973,

UNIVERSITY DE MONTPELLIER 


\section{PACIFIC JOURNAL OF MATHEMATICS}

\section{EDITORS}

RICHARD ARENS (Managing Editor) University of California

Los Angeles, California 90024

\section{R. A. Beaumont}

University of Washington Seattle, Washington 98105
J. DugundJI Department of Mathematics University of Southern Californıa Los Angeles, California 90007

D. Gilbarg aNd J. Milgram Stanford University Stanford, California 94305

\section{ASSOCIATE EDITORS}

E. F. BeCKENBACH

B. H. NeUmanN

F. Wolf

K. YoSHIDA

\section{SUPPORTING INSTITUTIONS}

UNIVERSITY OF BRITISH COLUMBIA CALIFORNIA INSTITUTE OF TECHNOLOGY UNIVERSITY OF CALIFORNIA MONTANA STATE UNIVERSITY UNIVERSITY OF NEVADA NEW MEXICO STATE UNIVERSITY OREGON STATE UNIVERSITY UNIVERSITY OF OREGON OSAKA UNIVERSITY
UNIVERSITY OF SOUTHERN CALIFORNIA STANFORD UNIVERSITY UNIVERSITY OF TOKYO UNIVERSITY OF UTAH WASHINGTON STATE UNIVERSITY UNIVERSITY OF WASHINGTON AMERICAN MATHEMATICAL SOCIETY NAVAL WEAPONS CENTER 


\section{Pacific Journal of Mathematics \\ Vol. 59, No. 1 \\ May, 1975}

Shashi Prabha Arya and M. K. Singal, More sum theorems for topological

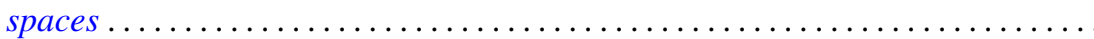

Goro Azumaya, F. Mbuntum and Kalathoor Varadarajan, On M-projective and

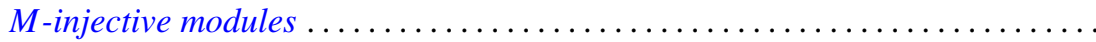

Kong Ming Chong, Spectral inequalities involving the infima and suprema of

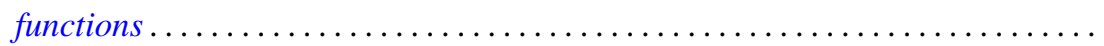

Alan Hetherington Durfee, The characteristic polynomial of the monodromy .......

Emilio Gagliardo and Clifford Alfons Kottman, Fixed points for orientation

preserving homeomorphisms of the plane which interchange two points ......

Raymond F. Gittings, Finite-to-one open maps of generalized metric spaces .......

Andrew M. W. Glass, W. Charles (Wilbur) Holland Jr. and Stephen H. McCleary,

$a^{*}$-closures of completely distributive lattice-ordered groups .............

Matthew Gould, Endomorphism and automorphism structure of direct squares of

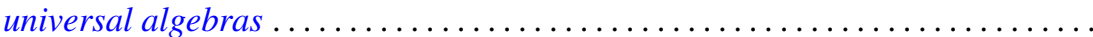

R. E. Harrell and Les Andrew Karlovitz, On tree structures in Banach spaces .....

Julien O. Hennefeld, Finding a maximal subalgebra on which the two Arens

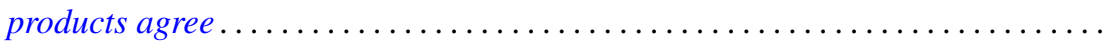

William Francis Keigher, Adjunctions and comonads in differential algebra .......

Robert Bernard Kelman, A Dirichlet-Jordan theorem for dual trigonometric

series

Allan Morton Krall, Stieltjes differential-boundary operators. III. Multivalued operators-linear relations...

Hui-Hsiung Kuo, On Gross differentiation on Banach spaces .

Tom Louton, A theorem on simultaneous observability ...

Kenneth Mandelberg, Amitsur cohomology for certain extensions of rings of algebraic integers.

Coy Lewis May, Automorphisms of compact Klein surfaces with boundary . . .

Peter A. McCoy, Generalized axisymmetric elliptic functions .

211

Muril Lynn Robertson, Concerning Siu's method for solving $y^{\prime}(t)=F(t$, $y(g(t)))$. .

Richard Lewis Roth, On restricting irreducible characters to normal

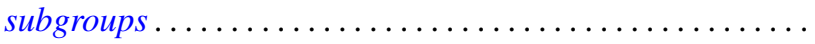

Albert Oscar Shar, $P$-primary decomposition of maps into an $H$-space .

Kenneth Barry Stolarsky, The sum of the distances to certain pointsets on the unit circle.

Bert Alan Taylor, Components of zero sets of analytic functions in $C^{2}$ in the unit ball or polydisc

Michel Valadier, Convex integrands on Souslin locally convex spaces ...

Januario Varela, Fields of automorphisms and derivations of $C$

Arnold Lewis Villone, A class of symmetric differential operators with deficiency

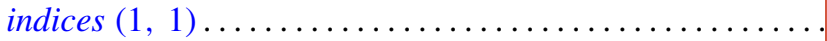

